\title{
Entropy on Riemann Surfaces and the Jacobians of Finite Covers
}

\section{Citation}

McMullen, Curtis T. Forthcoming. Entropy on Riemann surfaces and the Jacobians of finite covers. Commentarii Mathematici Helvetici.

\section{Permanent link}

http://nrs.harvard.edu/urn-3:HUL.InstRepos:9918807

\section{Terms of Use}

This article was downloaded from Harvard University's DASH repository, and is made available under the terms and conditions applicable to Open Access Policy Articles, as set forth at http:// nrs.harvard.edu/urn-3:HUL.InstRepos:dash.current.terms-of-use\#OAP

\section{Share Your Story}

The Harvard community has made this article openly available.

Please share how this access benefits you. Submit a story. 


\title{
Entropy on Riemann surfaces and the Jacobians of finite covers
}

\author{
Curtis T. McMullen
}

20 June, 2010

\begin{abstract}
This paper characterizes those pseudo-Anosov mappings whose entropy can be detected homologically by taking a limit over finite covers. The proof is via complex-analytic methods. The same methods show the natural map $\mathcal{M}_{g} \rightarrow \prod \mathcal{A}_{h}$, which sends a Riemann surface to the Jacobians of all of its finite covers, is a contraction in most directions.
\end{abstract}

\section{Contents}

$1 \quad$ Introduction . . . . . . . . . . . . . . . . . 1

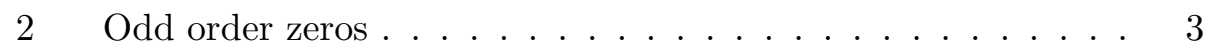

3 Siegel space . . . . . . . . . . . . . . . . 3

4 Teichmüller space . . . . . . . . . . . . . 6

5 Contraction .................... 7

6 Conclusion ....................... 8

A The hyperbolic metric via Jacobians of finite covers . . . . . 9

Research supported in part by the NSF.

2000 Mathematics Subject Classification: 32G15, 37E30. 


\section{Introduction}

Let $f: S \rightarrow S$ be a pseudo-Anosov mapping on a surface of genus $g$ with $n$ punctures. It is well-known that the topological entropy $h(f)$ is bounded below in terms of the spectral radius of $f^{*}: H^{1}(S, \mathbb{C}) \rightarrow H^{1}(S, \mathbb{C})$; we have

$$
\log \rho\left(f^{*}\right) \leq h(f) .
$$

If we lift $f$ to a map $\widetilde{f}: \widetilde{S} \rightarrow \widetilde{S}$ on a finite cover of $S$, then its entropy stays the same but the spectral radius of the action on homology can increase. We say the entropy of $f$ can be detected homologically if

$$
h(f)=\sup \log \rho\left(\widetilde{f}: H^{1}(\widetilde{S}) \rightarrow H^{1}(\widetilde{S})\right),
$$

where the supremum is taken over all finite covers to which $f$ lifts.

In this paper we will show:

Theorem 1.1 The entropy of a pseudo-Anosov mapping $f$ can be detected homologically if and only if the invariant foliations of $f$ have no odd-order singularities in the interior of $S$.

The proof is via complex analysis. Hodge theory provides a natural embedding $\mathcal{M}_{g} \rightarrow \mathcal{A}_{g}$ from the moduli space of Riemann surfaces into the moduli space of Abelian varieties, sending $X$ to its Jacobian. Any characteristic covering map from a surface of genus $h$ to a surface of genus $g$, branched over $n$ points, provides a similar map

$$
\mathcal{M}_{g, n} \rightarrow \mathcal{M}_{h} \rightarrow \mathcal{A}_{h}
$$

It is known that the hyperbolic metric on a Riemann surface $X$ can be reconstructed using the metrics induced from the Jacobians of its finite covers ([Kaz]; see the Appendix). Similarly, it is natural to ask if the Teichmüller metric on $\mathcal{M}_{g, n}$ can be recovered from the Kobayashi metric on $\mathcal{A}_{h}$, by taking the limit over all characteristic covers $\mathcal{C}_{g, n}$. We will show such a construction is impossible.

Theorem 1.2 The natural map $\mathcal{M}_{g, n} \rightarrow \prod_{\mathcal{C}_{g, n}} \mathcal{A}_{h}$ is not an isometry for the Kobayashi metric, unless $\operatorname{dim} \mathcal{M}_{g, n}=1$.

It is an open problem to determine if the Kobayashi and Carathéodory metrics on moduli space coincide when $\operatorname{dim} \mathcal{M}_{g, n}>1$ (see e.g. [FM, Prob 5.1]). An equivalent problem is to determine if Teichmüller space embeds holomorphically and isometrically into a (possibly infinite) product of bounded 
symmetric domains. Theorem 1.2 provides some support for a negative answer to this question.

Here is a more precise version of Theorem 1.2, stated in terms of the lifted map

$$
\mathcal{T}_{g, n} \rightarrow \mathcal{T}_{h} \stackrel{J}{\rightarrow} \mathfrak{H}_{h}
$$

from Teichmüller space to Siegel space determined by a finite cover.

Theorem 1.3 Suppose the Teichmüller mapping between a pair of distinct points $X, Y \in \mathcal{T}_{g, n}$ comes from a quadratic differential with an odd order zero. Then

$$
\sup d(J(\tilde{X}), J(\tilde{Y}))<d(X, Y),
$$

where the supremum is taken over all compatible finite covers of $X$ and $Y$.

Conversely, if the Teichmüller map from $X$ to $Y$ has only even order singularities, then there is a double cover such that $d(J(\widetilde{X}), J(\widetilde{Y}))=d(X, Y)$ (cf. [Kra]). In particular, the complex geodesics generated by squares of holomorphic 1-forms map isometrically into $\mathcal{A}_{g}$. The only directions contracted by the map $\mathcal{M}_{g} \rightarrow \prod \mathcal{A}_{h}$ are those identified by Theorem 1.3.

Theorem 1.1 follows from Theorem 1.3 by taking $X$ and $Y$ to be points on the Teichmüller geodesic stabilized by the mapping-class $f$. It would be interesting to find a direct topological proof of Theorem 1.1.

As a sample application, let $\beta \in B_{n}$ be a pseudo-Anosov braid whose monodromy map $f: S \rightarrow S$ (on the $n$-times punctured plane) has an odd order singularity. Then Theorem 1.1 implies the image of $\beta$ under the Burau representation satisfies

$$
\log \sup _{|q|=1} \rho(B(q))<h(f)
$$

Indeed, $\rho(B(q))$ at any $d$-th root of unit is bounded by $\rho\left(\widetilde{f}^{*}\right)$ on a $\mathbb{Z} / d$ cover $S$ [Mc2]. This improves a result in [BB]. Similar statements hold for other homological representations of the mapping-class group.

Notes and references. For $C^{\infty}$ diffeomorphisms of a compact smooth manifold, one has $h(f) \geq \log \sup _{i} \rho\left(f^{*} \mid H^{i}(X)\right)$ [Ym], and equality holds for holomorphic maps on Kähler manifolds [Gr]. The lower bound $h(f) \geq$ $\log \rho\left(f^{*} \mid H^{1}(X)\right)$ also holds for homeomorphisms [Mn]. For more on pseudoAnosov mappings, see e.g. [FLP], [Bers] and [Th].

A proof that the inclusion of $\mathcal{T}_{g, n}$ into universal Teichmüller space is a contraction, based on related ideas, appears in [Mc1]. 


\section{Odd order zeros}

We begin with an analytic result, which describes how well a monomial $z^{k}$ of odd order can be approximated by the square of an analytic function.

Theorem 2.1 Let $k \geq 1$ be odd, and let $f(z)$ be a holomorphic function on the unit disk $\Delta$ such that $\int|f(z)|^{2}=1$. Then

$$
\left|\int_{\Delta} f(z)^{2}\left(\frac{\bar{z}}{|z|}\right)^{k}\right| \leq C_{k}=\frac{\sqrt{k+1} \sqrt{k+3}}{k+2}<1 .
$$

Here the integral is taken with respect to Lebesgue measure on the unit disk.

Proof. Consider the orthonormal basis $e_{n}(z)=a_{n} z^{n}, n \geq 0, a_{n}=$ $\sqrt{n+1} / \sqrt{\pi}$, for the Bergman space $L_{\alpha}^{2}(\Delta)$ of analytic functions on the disk with $\|f\|_{2}^{2}=\int|f(z)|^{2}<\infty$. With respect to this basis, the nonzero entries in the matrix of the symmetric bilinear form $Z(f, g)=\int f(z) g(z) \bar{z}^{k} /|z|^{k}$ are given by

$$
Z\left(e_{n}, e_{k-n}\right)=a_{n} a_{k-n} \int_{\Delta}|z|^{k}=\frac{2 \sqrt{n+1} \sqrt{k-n+1}}{k+2} .
$$

In particular, $Z\left(e_{i}, e_{i}\right)=0$ for all $i$ (since $k$ is odd), and $Z\left(e_{i}, e_{j}\right)=0$ for all $i, j>k$.

Note that the ratio above is less than one, by the inequality between the arithmetic and geometric means, and it is maximized when $n<k / 2<$ $n+1$. Thus the maximum of $|Z(f, f)| /\|f\|^{2}$ over $L_{\alpha}^{2}(\Delta)$ is achieved when $f=e_{n}+e_{n+1}, n=(k-1) / 2$, at which point it is given by $C_{k}$.

\section{$3 \quad$ Siegel space}

In this section we describe the Siegel space of Hodge structures on a surface $S$, and its Kobayashi metric.

Hodge structures. Let $S$ be a closed, smooth, oriented surface of genus g. Then $H^{1}(S)=H^{1}(S, \mathbb{C})$ carries a natural involution $C(\alpha)=\bar{\alpha}$ fixing $H^{1}(S, \mathbb{R})$, and a natural Hermitian form

$$
\langle\alpha, \beta\rangle=\frac{\sqrt{-1}}{2} \int_{S} \alpha \wedge \bar{\beta}
$$


of signature $(g, g)$. A Hodge structure on $H^{1}(S)$ is given by an orthogonal splitting

$$
H^{1}(S)=V^{1,0} \oplus V^{0,1}
$$

such that $V^{1,0}$ is positive-definite and $V^{0,1}=C\left(V^{1,0}\right)$. We have a natural norm on $V^{1,0}$ given by $\|\alpha\|^{2}=\langle\alpha, \alpha\rangle$.

The set of all possible Hodge structures forms the Siegel space $\mathfrak{H}(S)$. To describe this complex symmetric space in more detail, fix a splitting $H^{1}(S)=W^{1,0} \oplus W^{0,1}$. Then for any other Hodge structure $V^{1,0} \oplus V^{0,1}$, there is a unique operator

$$
Z: W^{1,0} \rightarrow W^{0,1}
$$

such that $V^{1,0}=(I+Z)\left(W^{1,0}\right)$. This means $V^{1,0}$ coincides with the graph of $Z$ in $W^{1,0} \oplus W^{0,1}$.

The operator $Z$ is determined uniquely by the associated bilinear form

$$
Z(\alpha, \beta)=\langle\alpha, C Z(\beta)\rangle
$$

on $W^{1,0}$, and the condition that $V^{1,0} \oplus V^{0,1}$ is a Hodge structure translates into the conditions:

$$
Z(\alpha, \beta)=Z(\beta, \alpha) \text { and }|Z(\alpha, \alpha)|<1 \text { if }\|\alpha\|=1 .
$$

Since the second inequality above is an open condition, the tangent space at the base point $p \sim W^{1,0} \oplus W^{0,1}$ is given by

$$
\mathrm{T}_{p} \mathfrak{H}(S)=\left\{\text { symmetric bilinear maps } Z: W^{1,0} \times W^{1,0} \rightarrow \mathbb{C}\right\} .
$$

Comparison maps. Any Hodge structure on $H^{1}(S)$ determines an isomorphism

$$
V^{1,0} \cong H^{1}(S, \mathbb{R})
$$

sending $\alpha$ to $\operatorname{Re}(\alpha)=(\alpha+C(\alpha)) / 2$. Thus $H^{1}(S, \mathbb{R})$ inherits a norm and a complex structure from $V^{1,0}$.

Put differently, (3.2) gives a marking of $V^{1,0}$ by $H^{1}(S, \mathbb{R})$. By composing one marking with the inverse of another, we obtain the real-linear comparison map

$$
T=(I+Z)(I+C Z)^{-1}: W^{1,0} \rightarrow V^{1,0}
$$

between any pair of Hodge structures. It is characterized by $\operatorname{Re}(\alpha)=$ $\operatorname{Re}(T(\alpha))$. 
Symmetric matrices. The classical Siegel domain is given by

$$
\mathfrak{H}_{g}=\left\{Z \in \mathrm{M}_{g}(\mathbb{C}): Z_{i j}=Z_{j i} \text { and } I-Z \bar{Z} \gg 0\right\} .
$$

(cf. [Sat, Ch. II.7]). It is a convex, bounded symmetric domain in $\mathbb{C}^{N}$, $N=g(g+1) / 2$. The choice of an orthonormal basis for $W^{1,0}$ gives an isomorphism $Z \mapsto Z\left(\omega_{i}, \omega_{j}\right)$ between $\mathfrak{H}(S)$ and $\mathfrak{H}_{g}$, sending the basepoint $p$ to zero.

The Kobayashi metric. Let $\Delta \subset \mathbb{C}$ denote the unit disk, equipped with the metric $|d z| /\left(1-|z|^{2}\right)$ of constant curvature -4 . The Kobayashi metric on $\mathfrak{H}(S)$ is the largest metric such that every holomorphic map $f: \Delta \rightarrow \mathfrak{H}(S)$ satisfies $\|D f(0)\| \leq 1$. It determines both a norm on the tangent bundle and a distance function on pairs of points $[\mathrm{Ko}]$.

Proposition 3.1 The Kobayashi norm on $\mathrm{T}_{p} \mathfrak{H}(S)$ is given by

$$
\|Z\|_{K}=\sup \{Z(\alpha, \alpha) \mid:\|\alpha\|=1\},
$$

and the Kobayashi distance is given in terms of the comparison map (3.3) by

$$
d\left(V^{1,0}, W^{1,0}\right)=\log \|T\|
$$

Proof. Choosing a suitable orthonormal basis for $W^{1,0}$, we can assume that

$$
Z\left(\omega_{i}, \omega_{j}\right)=\lambda_{i} \delta_{i j}
$$

with $\lambda_{1} \geq \lambda_{2} \geq \cdots \lambda_{g} \geq 0$. Since $\mathfrak{H}_{g}$ is a convex symmetric domain, the Kobayashi norm at the origin and the Kobayashi distance satisfy

$$
\|Z\|_{K}=r \quad \text { and } \quad d(0, Z)=\frac{1}{2} \log \frac{1+r}{1-r},
$$

where $r=\inf \left\{s>0: Z \in s \mathfrak{H}_{g}\right\}$ (see $\left.[\mathrm{Ku}]\right)$. But clearly $r=\lambda_{1}=$ $\sup |Z(\alpha, \alpha)| /\|\alpha\|^{2}$, and by (3.3), we have

$$
\|T\|^{2}=\left\|T\left(\sqrt{-1} \omega_{1}\right)\right\|^{2}=\left\|\frac{\omega_{1}}{1-\lambda_{1}}+\frac{\lambda_{1} \bar{\omega}_{1}}{1-\lambda_{1}}\right\|^{2}=\frac{1+\lambda_{1}}{1-\lambda_{1}},
$$

which gives the expressions above. 


\section{Teichmüller space}

This section gives a functorial description of the derivative of the map from Teichmüller space to Siegel space.

Markings. Let $\bar{S}$ be a compact oriented surface of genus $g$, and let $S \subset \bar{S}$ be a subsurface obtained by removing $n$ points.

Let $\operatorname{Teich}(S) \cong \mathcal{T}_{g, n}$ denote the Teichmüller space of Riemann surfaces marked by $S$. A point in $\operatorname{Teich}(S)$ is specified by a homeomorphism $f$ : $S \rightarrow X$ to a Riemann surface of finite type. This means there is a compact Riemann surface $\bar{X} \supset X$ and an extension of $f$ to a homeomorphism $\bar{f}$ : $\bar{S} \rightarrow \bar{X}$.

Metrics. Let $Q(X)$ denote the space of holomorphic quadratic differentials on $X$ such that

$$
\|q\|_{X}=\int_{X}|q|<\infty
$$

There is a natural pairing $(q, \mu) \mapsto \int_{X} q \mu$ between the space $Q(X)$ and the space $M(X)$ of $L^{\infty}$-measurable Beltrami differentials $\mu$. The tangent and cotangent spaces to Teichmüller space at $X$ are isomorphic to $M(X) / Q(X)^{\perp}$ and $Q(X)$ respectively.

The Teichmüller and Kobayashi metrics on Teich $(S)$ coincide [Roy1], [Hub, Ch. 6]. They are given by the norm

$$
\|\mu\|_{T}=\sup \left\{\left|\int q \mu\right|:\|q\|_{X}=1\right\}
$$

on the tangent space at $X$; the corresponding distance function

$$
d(X, Y)=\inf \frac{1}{2} \log K(\phi)
$$

measures the minimal dilatation $K(\phi)$ of a quasiconformal map $\phi: X \rightarrow Y$ respecting their markings.

Hodge structure. The periods of holomorphic 1-forms on $X$ serve as classical moduli for $X$. From a modern perspective, these periods give a map

$$
J: \operatorname{Teich}(S) \rightarrow \mathfrak{H}(\bar{S}) \cong \mathfrak{H}_{g},
$$

sending $X$ to the Hodge structure

$$
H^{1}(\bar{S}) \cong H^{1}(\bar{X}) \cong H^{1,0}(\bar{X}) \oplus H^{0,1}(\bar{X}) .
$$

Here the first isomorphism is provided by the marking $\bar{f}: \bar{S} \rightarrow \bar{X}$. We also have a natural isomorphism between $H^{1,0}(\bar{X})$ and the space of holomorphic 
1-forms $\Omega(\bar{X})$. The image $J(X)$ encodes the complex analytic structure of the Jacobian variety $\operatorname{Jac}(\bar{X})=\Omega(\bar{X})^{*} / H_{1}(\bar{X}, \mathbb{Z})$. (It is does not depend on the location of the punctures of $X$.)

Proposition 4.1 The derivative of the period map sends $\mu \in M(X)$ to the quadratic form $Z=D J(\mu)$ on $\Omega(\bar{X})$ given by

$$
Z(\alpha, \beta)=\int_{\bar{X}} \alpha \beta \mu
$$

This is a basis-free reformulation of Ahlfors' variational formula [Ah, §5]; see also [Ra], [Roy2] and [Kra, Prop. 1]. Note that $\alpha \beta \in Q(X)$.

\section{Contraction}

This section brings finite covers into play, and establishes a uniform estimate for contraction of the mapping $\mathcal{T}_{g, n} \rightarrow \mathcal{T}_{h} \rightarrow \mathfrak{H}_{h}$.

Jacobians of finite covers. A finite connected covering space $S_{1} \rightarrow S_{0}$ determines a natural map

$$
P: \operatorname{Teich}\left(S_{0}\right) \rightarrow \operatorname{Teich}\left(S_{1}\right)
$$

sending each Riemann surface to the corresponding covering space $X_{1} \rightarrow X_{0}$. By taking the Jacobian of $X_{1}$, we obtain a map $J \circ P: \operatorname{Teich}\left(S_{0}\right) \rightarrow \mathfrak{H}\left(\bar{S}_{1}\right)$.

Let $q_{0} \in Q\left(X_{0}\right)$ be a holomorphic quadratic differential with a zero of odd order $k$, say at $p \in X_{0}$. Let $\mu=\bar{q}_{0} /\left|q_{0}\right| \in M\left(X_{0}\right)$; then $\|\mu\|_{T}=1$. Let $\pi: X_{1} \rightarrow X_{0}$ denote the natural covering map, and let $q_{1}=\pi^{*}\left(q_{0}\right)$.

We will show that $J\left(X_{1}\right)$ cannot change too rapidly under the unit deformation $\mu$ of $X_{0}$. Indeed, if $J\left(X_{1}\right)$ were to move at nearly unit speed, then $\pi^{*}(\mu)=\bar{q}_{1} /\left|q_{1}\right|$ would pair efficiently with $\alpha^{2}$ for some unit-norm $\alpha \in \Omega\left(\bar{X}_{1}\right)$, which is impossible because of the many odd-order zeros of $q_{1}$.

To make a quantitative estimate, choose a holomorphic chart $\phi:(\Delta, 0) \rightarrow$ $\left(X_{0}, p\right)$ such that $\phi^{*}(\mu)=z^{k} /|z|^{k} d \bar{z} / d z$. Let $U=\phi(\Delta)$, and let

$$
m(U)=\inf \left\{\|q\|_{U}: q \in Q\left(X_{0}\right),\|q\|_{X}=1\right\} .
$$

(Here $\|q\|_{U}=\int_{U}|q|$.) Since $Q\left(X_{0}\right)$ is finite-dimensional, we have $m(U)>0$.

Theorem 5.1 The image $Z$ of the vector $[\mu]$ under the derivative of $J \circ P$ satisfies

$$
\|Z\|_{K} \leq \delta<1=\|\mu\|_{T}
$$

where $\delta=\max \left(1 / 2,1-\left(1-C_{k}\right) m(U) / 2\right)$ does not depend on the finite cover $S_{1} \rightarrow S_{0}$. 
Proof. The derivative of $P$ sends $\mu$ to $\pi^{*}(\mu)$. By Proposition 3.1, to show $\|Z\|_{K} \leq \delta$ it suffices to show that

$$
|Z(\alpha, \alpha)|=\left|\int_{X_{1}} \alpha^{2} \pi^{*} \mu\right| \leq \delta
$$

for all $\alpha \in \Omega\left(\bar{X}_{1}\right)$ with $\left\|\alpha^{2}\right\|_{X_{1}}=1$. Setting $q=\pi_{*}\left(\alpha^{2}\right)$, we also have

$$
|Z(\alpha, \alpha)|=\left|\int_{X_{0}} q \mu\right| \leq\|q\|_{X_{0}}
$$

so the proof is complete if $\|q\|_{X_{0}} \leq 1 / 2$. Thus we may assume that

$$
\left\|\alpha^{2}\right\|_{V} \geq\|q\|_{U} \geq m(U)\|q\|_{X_{0}} \geq m(U) / 2,
$$

where $V=\pi^{-1}(U)=\bigcup_{1}^{d} V_{i}$ is a finite union of disjoint disks. Using the coordinate charts $V_{i} \cong U \cong \Delta$ and Theorem 2.1, we find that on each of these disks we have

$$
\left|\int_{V_{i}} \alpha^{2} \pi^{*}(\mu)\right|=\left|\int_{\Delta} \alpha(z)^{2}\left(\frac{z}{|z|}\right)^{k}\right| \leq C_{k}\left\|\alpha^{2}\right\|_{V_{i}} .
$$

Summing these bounds and using the fact that $\left\|\alpha^{2}\right\|_{\left(X_{1}-V\right)}+\left\|\alpha^{2}\right\|_{V}=1$, we obtain

$$
\left|\int_{X_{1}} \alpha^{2} \pi^{*}(\mu)\right| \leq\left\|\alpha^{2}\right\|_{\left(X_{1}-V\right)}+C_{k}\left\|\alpha^{2}\right\|_{V} \leq 1-\frac{\left(1-C_{k}\right) m(U)}{2} \leq \delta .
$$

\section{Conclusion}

It is now straightforward to establish the results stated in the Introduction. Proof of Theorems 1.3. Assume the Beltrami coefficient of the Teichmüller mapping between $X, Y \in \mathcal{T}_{g, n}$ has the form $\mu=k \bar{q} / q$, where $q \in Q(X)$ has an odd order zero. Then the same is true for the tangent vectors to the Teichmüller geodesic $\gamma$ joining $X$ to $Y$. Theorem 5.1 then implies that $\left.D(J \circ P)\right|_{\gamma}$ is contracting by a factor $\delta<1$ independent of $P$, and therefore

$$
d(J \circ P(X), J \circ P(Y))=d(J(\widetilde{X}), J(\widetilde{Y}))<\delta \cdot d(X, Y) .
$$


Proof of Theorem 1.2. The contraction of $\mathcal{M}_{g, n} \rightarrow \prod_{\mathcal{C}_{g, n}} \mathcal{A}_{h}$ in some directions is immediate from the uniformity of the bound in Theorem 1.3, using the fact that the Kobayashi metric on a product is the sup of the Kobayashi metrics on each term, and that there exist $q \in Q(X)$ with simple zeros whenever $X \in \mathcal{M}_{g, n}$ and $\operatorname{dim} \mathcal{M}_{g, n}>1$.

Proof of Theorem 1.1. Let $f: S_{0} \rightarrow S_{0}$ be a pseudo-Anosov mapping. If $f$ has only even order singularities, then its expanding foliation is locally orientable, and hence there is a double cover $\widetilde{S} \rightarrow \widetilde{S}$ such that $\log \rho\left(\widetilde{f}^{*}\right)=$ $h(f)$.

Now suppose $f$ has an odd-order singularity. Let $X_{0} \in \operatorname{Teich}\left(S_{0}\right)$ be a point on the Teichmüller geodesic stabilized by the action of $f$ on Teich $\left(S_{0}\right)$. Then $d\left(f \cdot X_{0}, X_{0}\right)=h(f)>0$ (see e.g. [FLP] and [Bers]).

Let $\widetilde{f}: S_{1} \rightarrow S_{1}$ be a lift of $f$ to a finite covering of $S_{0}$, and let $X_{1}=P\left(X_{0}\right) \in \operatorname{Teich}\left(S_{1}\right)$. Using the marking of $X_{1}$ and the isomorphism $H^{1}\left(X_{1}, \mathbb{R}\right) \cong H^{1,0}\left(X_{1}\right)$, we obtain a commutative diagram

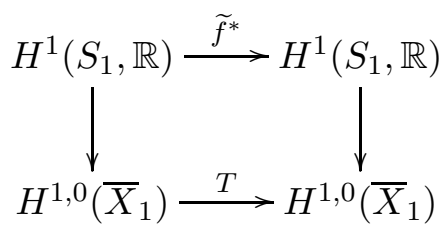

where $T$ is the comparison map between $J\left(X_{1}\right)$ and $J\left(\widetilde{f} \cdot X_{1}\right)$ (see equation (3.3)). Then Theorem 1.3 and Proposition 3.1 yield the bound

$$
\log \rho\left(\tilde{f}^{*}\right) \leq \log \|T\|=d\left(J\left(X_{1}\right), \tilde{f} \cdot J\left(X_{1}\right)\right) \leq \delta d\left(X_{0}, f \cdot X_{0}\right)=\delta h(f),
$$

where $\delta<1$ does not dependent on the finite covering $S_{1} \rightarrow S_{0}$. Consequently, sup $\log \rho\left(\tilde{f}^{*}\right)<h(f)$.

\section{A The hyperbolic metric via Jacobians of finite covers}

Let $X=\Delta / \Gamma$ be a compact Riemann surface, presented as a quotient of the unit disk by a Fuchsian group $\Gamma$. Let $Y_{n} \rightarrow X$ be an ascending sequence of finite Galois covers which converge to the universal cover, in the sense that

$$
Y_{n}=\Delta / \Gamma_{n}, \quad \Gamma \supset \Gamma_{1} \supset \Gamma_{2} \supset \Gamma_{3} \cdots, \quad \text { and } \bigcap \Gamma_{i}=\{e\} .
$$


The Bergman metric on $Y_{n}$ (defined below) is invariant under automorphisms, so it descends to a metric $\beta_{n}$ on $X$. This appendix gives a short proof of:

Theorem A.1 (Kazhdan) The Bergman metrics inherited from the finite Galois covers $Y_{n} \rightarrow X$ converge to a multiple of the hyperbolic metric; more precisely, we have

$$
\beta_{n} \rightarrow \frac{\lambda_{X}}{2 \sqrt{\pi}}
$$

uniformly on $X$.

The argument below is based on [Kaz, §3]; for another, somewhat more technical approach, see $[\mathrm{Rh}]$.

Metrics. We begin with some definitions. Let $\Omega(X)$ denote the Hilbert space of holomorphic 1-forms on a Riemann surface $X$ such that

$$
\|\omega\|_{X}^{2}=\int_{X}|\omega|^{2}<\infty .
$$

The area form of the Bergman metric on $X$ is given by

$$
\beta_{X}^{2}=\sum\left|\omega_{i}\right|^{2}
$$

where $\left(\omega_{i}\right)$ is any orthonormal basis of $\Omega(X)$. Equivalently, the Bergman length of a tangent vector $v \in \mathrm{T} X$ is given by

$$
\left\langle\beta_{X}, v\right\rangle=\sup _{\omega \neq 0} \frac{|\omega(v)|}{\|\omega\|_{X}} .
$$

This formula shows that inclusions are contracting: if $Y$ is a subdomain of $X$, then $\beta_{Y} \geq \beta_{X}$.

Now suppose $X$ is a compact surface of genus $g>0$. Then (A.2) shows its Bergman area is given by

$$
\int_{X} \beta_{X}^{2}=\operatorname{dim} \Omega(X)=g
$$

In this case $\beta_{X}$ is also the pullback, via the Abel-Jacobi map, of the natural Kähler metric on the Jacobian of $X$.

Finally suppose $X=\Delta / \Gamma$. Then the hyperbolic metric of constant curvature -1 ,

$$
\lambda_{\Delta}=\frac{2|d z|}{1-|z|^{2}}
$$


descends to give the hyperbolic metric $\lambda_{X}$ on $X$. Using the fact that $\|d z\|_{\Delta}=$ $\pi$, it is easy to check that $4 \pi \beta_{\Delta}^{2}=\lambda_{\Delta}^{2}$.

Proof of Theorem A.1. We will regard the Bergman metric $\beta_{n}$ on $Y_{n}$ as a $\Gamma_{n}$-invariant metric on $\Delta$. It suffices to show that $\beta_{n} / \beta_{\Delta} \rightarrow 1$ uniformly on $\Delta$.

Let $g$ and $g_{n}$ denote the genus of $X$ and $Y_{n}$ respectively, and let $d_{n}$ denote the degree of $Y_{n} / X$; then $g_{n}-1=d_{n}(g-1)$. By (A.1), the injectivity radius of $Y_{n}$ tends to infinity. In particular, there is a sequence $r_{n} \rightarrow 1$ such that $\gamma\left(r_{n} \Delta\right)$ injects into $Y_{n}$ for any $\gamma \in \Gamma$. Since inclusions are contracting, this shows

$$
\beta_{n} \leq\left(1+\epsilon_{n}\right) \beta_{\Delta}
$$

where $\epsilon_{n} \rightarrow 0$.

Next, note that both $\beta_{n}$ and $\beta_{\Delta}$ are $\Gamma$-invariant, so they determine metrics on $X$. By (A.4), we have

$$
\int_{X} \beta_{n}^{2}=\frac{1}{d_{n}} \int_{Y_{n}} \beta_{n}^{2}=\frac{g_{n}}{d_{n}} \rightarrow(g-1)=\int_{X} \beta_{\Delta}^{2}
$$

(since $\int_{X} \lambda_{X}^{2}=2 \pi(2 g-2)$ by Gauss-Bonnet). Together with (A.5), this implies

$$
\int_{X}\left|\beta_{n}-\beta_{\Delta}\right|^{2} \rightarrow 0
$$

To show $\beta_{n} \rightarrow \beta_{\Delta}$ uniformly, consider any sequence $p_{n} \in \Delta$ and let $x \in[0,1]$ be a limit point of $\left(\beta_{n} / \beta_{\Delta}\right)\left(p_{n}\right)$. It suffices to show $x=1$.

Passing to a subsequence and using compactness of $X$, we can assume that $p_{n} \rightarrow p \in \Delta$ and that $\beta_{n}\left(p_{n}\right) \rightarrow x \beta_{\Delta}(p)$. By changing coordinates on $\Delta$, we can also assume $p=0$. By (A.6) we can find $q_{n} \rightarrow 0$ such that $\beta_{n}\left(q_{n}\right) \rightarrow \beta_{\Delta}(0)$. Then by (A.3), there exist $\Gamma_{n}$-invariant holomorphic 1forms $\omega_{n}(z) d z$ on $\Delta$ such that $\int_{Y_{n}}\left|\omega_{n}\right|^{2}=1$ and

$$
\left|\omega_{n}\left(q_{n}\right)\right|=\beta_{n}\left(q_{n}\right) \rightarrow \beta_{\Delta}(0)=\frac{|d z|}{\pi} .
$$

Since $\omega_{n}$ is holomorphic and $\int_{r_{n} \Delta}\left|\omega_{n}\right|^{2}<1$, the equation above easily implies that $\left|\omega_{n}\right| \rightarrow|d z| / \pi$ uniformly on compact subsets of $\Delta$. But we also have

$$
\beta_{n}\left(p_{n}\right) \geq\left|\omega_{n}\left(p_{n}\right)\right| \rightarrow \beta_{\Delta}(0)
$$

and thus $\beta_{n}\left(p_{n}\right) \rightarrow \beta_{\Delta}(0)$ and hence $x=1$. 


\section{References}

[Ah] L. Ahlfors. The complex analytic structure of the space of closed Riemann surfaces. In Analytic Functions, pages 45-66. Princeton Univ. Press, 1960.

[BB] G. Band and P. Boyland. The Burau estimate for the entropy of a braid. Preprint, 2008.

[Bers] L. Bers. An extremal problem for quasiconformal maps and a theorem by Thurston. Acta Math. 141(1978), 73-98.

[FLP] A. Fathi, F. Laudenbach, and V. Poénaru. Travaux de Thurston sur les surfaces. Astérisque, vol. 66-67, 1979.

[FM] A. Fletcher and V. Markovic. Infinite dimensional Teichmüller spaces. In A. Papadopoulos, editor, Handbook of Teichmüller Theory, volume II, pages 65-92. Eur. Math. Soc., 2009.

[Gr] M. Gromov. On the entropy of holomorphic maps. Enseign. Math. 49(2003), 217-235.

[Hub] J. H. Hubbard. Teichmüller Theory, vol. I. Matrix Editions, 2006.

[Kaz] D. A. Kazhdan. On arithmetic varieties. In Lie Groups and Their Representations (Proc. Summer School, Bolyai János Math. Soc., Budapest, 1971), pages 151-217. Halsted, 1975.

[Ko] S. Kobayashi. Hyperbolic Manifolds and Holomorphic Mappings. Marcel Dekker, Inc., 1970.

[Kra] I. Kra. The Carathéodory metric on abelian Teichmüller disks. J. Analyse Math. 40(1981), 129-143.

$[\mathrm{Ku}]$ Y. Kubota. On the Kobayashi and Carathéodory distances of bounded symmetric domains. Kodai Math. J. 12(1989), 41-48.

[Mn] A. Manning. Topological entropy and the first homology group. In Dynamical systems - Warwick 1974, volume 468 of Lecture Notes in Math., pages 185-190. Springer, 1975.

[Mc1] C. McMullen. Amenability, Poincaré series and quasiconformal maps. Invent. math. 97(1989), 95-127. 
[Mc2] C. McMullen. Braid groups and Hodge theory. Math. Ann., to appear.

[Ra] H. E. Rauch. A transcendental view of the space of algebraic Riemann surfaces. Bull. Amer. Math. Soc. 71(1965), 1-39.

[Rh] J. A. Rhodes. Sequences of metrics on compact Riemann surfaces. Duke Math. J. 72(1993), 725-738.

[Roy1] H. L. Royden. Automorphisms and isometries of Teichmüller space. In Advances in the Theory of Riemann Surfaces, pages 369-384. Princeton University Press, 1971.

[Roy2] H. L. Royden. Invariant metrics on Teichmüller space. In Contributions to Analysis, pages 393-399. Academic Press, 1974.

[Sat] I. Satake. Algebraic Structures of Symmetric Domains. Princeton University Press, 1980.

[Th] W. P. Thurston. On the geometry and dynamics of diffeomorphisms of surfaces. Bull. Amer. Math. Soc. 19(1988), 417-432.

[Ym] Y. Yomdin. Volume growth and entropy. Israel J. Math. 57(1987), 285-300.

\author{
Mathematics Department \\ HARVARD UNIVERSITY \\ 1 OXFord ST \\ CAMBridge, MA 02138-2901
}

